\title{
iCOPE with COVID-19: A Brief Telemental Health Intervention for Children and Adolescents during the COVID-19 Pandemic
}

Michelle S. Zepeda ${ }^{1}$, M.Sc., Stephanie Deighton ${ }^{1}$, M.Sc., Veronika Markova ${ }^{1}$, M.A., Joshua W. Madsen $^{1}$, Ph.D., R.Psych. Nicole Racine ${ }^{1}$, Ph.D., R.Psych.

Affiliations: ${ }^{1}$ Department of Psychology, University of Calgary, Calgary, Alberta, Canada.

Short title: Telemental health for children and adolescents during COVID-19.

Tables $=2(1$ in Supplementary Material $)$

Figures $=0$

\section{Author Note}

We have no known conflict of interest to disclose.

Acknowledgements: We would like to thank the families and children who received the iCOPE with COVID-19 protocol, and all of the clinical psychology graduate students who delivered the intervention. We would also like to thank Dr. Melanie Badali, Dr. Ena Vukatana, Dr. Kyleigh Schraeder, and Keely McSpurren for their thoughtful feedback on early versions of the protocol.

Funding source: MSZ is supported by a SSHRC Doctoral Fellowship. NR is supported by a fellowship from Alberta Innovates. SD is supported by a CIHR Doctoral Award.

Address correspondence to: Nicole Racine, Ph.D, R.Psych, Department of Psychology

University of Calgary, Calgary, AB, T2N 1N4, Canada, Phone: 403-992-7869; Email:

nicole.racine2@ucalgary.ca 


\section{Highlights}

- A brief telemental health intervention (iCOPE with COVID-19) was developed for youth.

- The intervention was delivered at the outset of the COVID-19 pandemic.

- Child and adolescent anxiety decreased post-intervention compared to pre-intervention.

- Results suggest acceptance of iCOPE with COVID-19 with children and adolescents. 


\begin{abstract}
The COVID-19 pandemic has prompted unprecedented disruptions to the daily lives of children and adolescents worldwide, which has been associated with an increase of anxiety and depressive symptoms in youth. However, due to public health measures, in-person psychosocial care has been affected causing barriers to mental health care access. This study investigated the feasibility, acceptability and preliminary effectiveness of iCOPE with COVID-19, a brief telemental health intervention for children and adolescents to address anxiety symptoms. Sessions were provided exclusively using videoconferencing technology. Feasibility and acceptability were measured with client satisfaction data. The main outcome measure for effectiveness was anxiety symptom severity measured using the Screen for Child Anxiety and Related Disorders (SCARED). Results indicated that the treatment was well accepted by participants. Significant reductions in anxiety were noted for social anxiety, and were observed to be trending towards a mean decrease for total anxiety. The findings suggest that this brief telemental health intervention focused on reducing anxiety related to COVID-19 is acceptable and feasible to children and adolescents. Future research using a large sample and with a longer follow-up period could inform whether symptom decreases are sustained over time.
\end{abstract}

Keywords: COVID-19, child, adolescent, telemental health, anxiety, cognitive-behavior therapy 


\section{iCOPE with COVID-19: A Brief Telemental Health Intervention for Children and Adolescents during the COVID-19 Pandemic}

As a result of the public health measures implemented as part of the COVID-19 pandemic, children and adolescents have experienced unprecedented disruptions to their daily lives and routines. In March 2020, emergency measures were enacted internationally to contain the spread of COVID-19, which effectively initiated the widespread interruptions to the lives of children and adolescents, including school closures, cancellation of extra-curricular activities, and reduced social interactions with peers, friends, and extended family (Lee, 2020). Furthermore, at the outset of the COVID-19 pandemic, children and adolescents experienced heightened fear and anxiety related to uncertainty about the future, their own health, and the health and well-being of their family (Smirni et al., 2020). Since the onset of the COVID-19 pandemic, emerging research has begun to elucidate the psychological impacts of the disruptions on the mental health of children and adolescents. Specifically, a rapid review of the literature examining the mental health of children and adolescents as a result of the COVID-19 pandemic observed an increase in depressive and anxiety symptoms in children and adolescents around the globe (Racine et al., 2020). Indeed, reports from China (Jiao, et al., 2020; Liu et al., 2020; Xie et al., 2020; Zhou et al., 2020), the United States (Rosen et al., 2020), and Canada (Cost et al., 2021) have all documented elevations in child and/or youth psychological distress. Despite the increase in mental health needs for children and youth during the COVID-19 pandemic, there were no known evidence-based interventions to specifically address pandemic-related fear and anxiety for children and youth. Furthermore, few mental health practitioners were providing services remotely via telemental health. Thus, driven by a community-based need to provide mental health supports to children and 
youth during the early months of the COVID-19 pandemic, the current study sought to develop and evaluate a brief, telemental health intervention to address this service need.

With the acute need for mental health support targeted at children and adolescents during the outset of the COVID-19 pandemic, two important practice gaps emerged. First, as a result of public health measures, psychosocial services could not be delivered in-person. Yet, very few practitioners were providing psychotherapy online prior to the pandemic and most providers had to rapidly pivot to delivering services remotely via telemental health (Madigan et al, 2021). While there was evidence to support the use of telemental health (Boydell et al., 2014; Calear \& Christensen, 2010; Hollis et al. 2017), very few interventions had pre-existing online resources (e.g., MoodGYM; Christensen \& Griffiths, 2005; SPARX; Merry et al. 2012), were easily adaptable for use with telemental health, or were designed to be delivered remotely (e.g., Cool Kids Program; McLoone et al., 2006; Coping Cat; Podell et al., 2010). Specifically, they lacked resources and activities that were engaging for children in an online format.

A second practice gap at the outset of the pandemic was that there were no brief child or adolescent-focused interventions that directly addressed fears related to a pandemic. While past research had found that internet-based cognitive behavioural therapy (ICBT) programs have been effective in preventing and reducing anxiety symptoms (Calear \& Christensen, 2010; March et al., 2009; Ye et al., 2014), depressive symptoms (e.g., Christensen \& Griffiths, 2005; Merry et al., 2012) and chronic pain (e.g., Law et al., 2012; Stinson et al., 2014) in youth populations, they did not include specific considerations required to address fears and anxieties in the context of a pandemic. That is, many of the fears and anxieties that accompanied the COVID-19 pandemic for children were different than those that would be explored in a typical child or adolescent-focused anxiety intervention. For example, many cognitive-behavioural interventions for anxiety for 
children involve challenging thoughts and fears that are unrealistic, and gradual exposure to a feared event (Albano \& Kendall, 2002). However, these approaches seemed less fitting as many aspects of the pandemic were initially unknown and represented a legitimate threat to health. Therefore, approaches that integrated coping strategies, relaxation strategies, and distress regulation were considered to be more optimal.

\section{Current Study}

At the time that the initial rapid spread of COVID-19 prompted the emergency public health measures across Canada, there were no brief, telemental health interventions developed specifically targeting both children and adolescents' anxiety and fear related to the COVID-19 pandemic. As such, with the growing need for mental health support, coupled with limited intervention options available to provide that support remotely we aimed to address this growing mental health need by developing an intervention that would be widely accessible and would target children and adolescents' increased distress related to COVID-19. Additionally, we sought to conduct a preliminary pilot evaluation using pre and post outcome measures of child and adolescent anxiety as well as to provide initial data on the acceptability and feasibility of the intervention.

\section{Method}

\section{Participants and Procedures}

A total of 27 children aged 6 to 12 years of age were included in the current study. All children resided in Alberta, Canada and were referred by parents and caregivers to the University of Calgary Psychology Clinic between April and September 2020. Children were recruited through social media (e.g., Facebook and Twitter), listserv and email advertisements, as well as advertisements through community organizations. Children were referred for a variety of 
presenting concerns including stress, anxiety, difficulty coping, and avoidance of social relationships, prompted by the COVID-19 pandemic. Although recruitment was initially targeted at children of healthcare workers, this was expanded to include all children in June 2020. Intake interviews were scheduled with all parents and their children, when appropriate (i.e., children were older than 7 years of age), to determine eligibility. Ineligibility criteria included: young age of child (i.e., under 6 years of age), presenting concerns not related to COVID-19 anxiety (e.g., distractibility or family trauma), and presenting concerns that were too severe for treatment via videoconferencing psychotherapy (i.e., suicidal ideation or non-suicidal self-injury behavior). A total of 16 children were deemed ineligible for the iCOPE with COVID-19 protocol, and were referred to other mental health services. If deemed eligible, after the initial intake, the child was scheduled for three weekly sessions with one of five clinical psychology graduate students. Due to the public health measures, the training clinic was physically closed to students and staff, as such, sessions were conducted remotely from a secure and private location in students' homes. The delivery of the iCOPE with COVID-19 intervention was supervised by a registered doctorallevel clinical psychologist. Electronic copies of the iCOPE with COVID-19 protocol were sent to the parents of eligible children following intake, for children's use during sessions.

Written informed consent was obtained from parents and limits of confidentiality were discussed with both parents, and child and adolescent participants prior to beginning the protocol. Ethical approval for this study was obtained from the University of Calgary Research Ethics Board (REB17-1077).

\section{Development of iCOPE with COVID-19}

The iCOPE with COVID-19 protocol consists of a three-session structure to target anxiety related to COVID-19. The treatment protocol was designed to be a brief telemental health 
intervention, integrating elements of various evidence-based approaches, including CognitiveBehavioral Therapy and Dialectical Behavior Therapy. The treatment was delivered during three weekly sessions. The goal of iCOPE with COVID-19 was to provide children with psychoeducation regarding emotions, and to teach strategies for managing difficult emotions related to COVID-19. The content for the three treatment sessions is outlined below. A copy of the therapist and treatment manuals are available free of charge by requesting a copy via email at PsyClinic@ucalgary.ca.

The iCOPE with COVID-19 manual was developed by two senior-level clinical psychology doctoral students at the University of Calgary (MZ, SD) under the supervision of a registered doctoral level clinical psychologis (NR). During the development phase, the protocol was shared for feedback from three doctoral-level psychologists, a stakeholder from a national mental health organization, and one youth stakeholder. Feedback from these consultations with regards to length, content, and visuals were integrated into the final version of the protocol.

\section{Session 1}

The first session of iCOPE with COVID-19 is focused on building rapport with the client, providing psychoeducation about COVID-19 and emotions, and practicing a relaxation strategy to manage anxiety. Specifically, the first activity was a series of alternating questions focused on building rapport between the client and the therapist. Next, the therapist provided the client with psychoeducation about COVID-19. For example, the protocol stated that "whenever anyone sneezes or coughs, there are tiny droplets that may stay in the air or stay on surfaces. When someone has COVID-19 those tiny droplets can spread to others if they get on surfaces like doorknobs and water bottles, and someone else touches them and then touches their mouth, nose or eyes. Someone can also get sick if they are very close to someone else who has COVID-19." 
Children were also taught that symptoms could range from mild to severe, and that most people who contract COVID-19 have mild symptoms. Children were encouraged to participate by brainstorming actions that they could take to reduce the spread of COVID-19 (e.g., washing hands, physical distancing and the use of masks). Following this, children were provided with psychoeducation regarding emotions, with an emphasis on the emotions related to COVID-19 such as anxiety, loss, fear, and disappointment. Here, therapists were encouraged to use the "whiteboard feature" on the remote therapy platform to have children identify the emotions that they have experienced since the onset of the COVID-19 pandemic. Lastly, children were taught diaphragmatic breathing, a common strategy taught in cognitive-behavioural therapy that has demonstrated effectiveness in reducing stress and negative affect (Ma et al., 2017). Therapists first modeled the skill and then children practiced during the session. Children were subsequently encouraged to practice diaphragmatic breathing over the following week as home-practice. They were provided with online videos to use as examples when practicing at home. Therapists and children then worked together to collaboratively identify three treatment goals. At the end of the session, therapists met briefly with parent(s) or caregiver(s) to review the content of the session.

\section{Session 2}

The second session focused on reviewing homework from Session 1 and identifying other strategies for emotion regulation. First, therapists reviewed "action" strategies to help manage and prevent the difficult emotions related to COVID-19. This list included reducing the amount of media exposure, staying socially connected to peers, and maintaining a routine. Children were encouraged to identify which strategies they might try over the following week. Next, children were presented with two new relaxation strategies: progressive muscle relaxation and guided imagery. Therapists practiced one or more of these strategies with the child in the session, based 
on child preference. Finally, using a cognitive-behavioural theoretical framework, children were provided with psychoeducation regarding thoughts and their connection to emotions and behaviours. The iCOPE with COVID-19 intervention protocol was designed to be used with children of differing ages and developmental levels. As such, therapists had the opportunity to choose one of two activities for their client. For older children, therapists were encouraged to guide the child through a cognitive challenging exercise "Switch That Thought", where children were taught how to switch an unhelpful thought to a helpful thought. Cognitive restructuring is a core component of several CBT interventions (Beck, 1976; Rapee, et al., 2000). During this activity, children were provided psychoeducation on what thoughts are, and they were encouraged to think of examples of different thoughts. Next, children were taught about unhelpful thoughts (i.e., thoughts that may not be true, or that get in the way of dealing with big feelings or problem solving), and helpful thoughts (i.e., thoughts that are more realistic and improve our functioning). Children and their therapist then read over examples of unhelpful (e.g., "I hate staying at home, it is so boring") and helpful thoughts (e.g., "staying at home is hard, but I am doing it to stay safe and I have good coping tools to help me") together, and children were encouraged to identify personal examples of both. Using a personal example of an unhelpful thought, children were supported in "switching" the unhelpful thought to a more helpful one.

With younger children, therapists taught children to externalize their thoughts and, if appropriate, challenge their externalized thought. For example, children were encouraged to draw a picture of their worry or anxious thought (e.g., worry bug or worry monster) and to challenge it. For homework, children were encouraged to practice some of the action, relaxation and thought strategies identified during the session over the following week. At the end of the session, therapists reviewed the session with the child's parent(s) or caregiver(s). 


\section{Session 3}

The third session was dedicated to helping children develop strategies to manage emotions by using mindfulness, present-moment strategies and dialectical behavioural strategies. Children were taught that emotions are like a rollercoaster that get "big and intense" but that also come down with time. Children were encouraged to use present-moment strategies to manage their intense emotions (such as paying close attention to their five senses). Next, children were asked to identify DISTRACT skills that they could use if their emotions became really intense. DISTRACT skills are a dialectical behaviour therapy skill that have been developed in previous research (Perepletchikova et al., 2011). These skills included: Do something else, Imagine pleasant events, Stop thinking about it, Think about something else, Remind yourself of positive experiences, Ask for help, Count your breaths, and Take a break (Perepletchikova et al., 2011). During this activity, children and therapists reviewed the description of the skills together on the screen, and children were encouraged to identify one personal example for each skill.

Children were then taught to identify the aspects of a situation that they have control over, and those that they do not. These were drawn on a "circle of control" diagram (See Figure 1). Using this diagram, children would identify the actions they are taking that are in their control and write these on the inside of the circle (e.g., maintaining social distance, wearing a mask, washing my hands). Children also identified things that were not in their control (e.g., when school will start back up again, whether my extra-curriculars will resume, if other people follow the public health rules) and documented these on the outside of the circle. Children were encouraged to do this with situations related to COVID-19, and then to focus their attention to the aspects of the situation that they can control. Afterward, therapists highlighted the positive consequences of helping behaviours and supported children in identifying people in the lives that they could help. 
At the end of the session, children were asked to identify three of the most helpful strategies they had learned over the course of the last three sessions. Similar to previous sessions, therapists reviewed the content of the sessions with parent(s) or caregiver(s) at the end of the final appointment.

\section{Additional Resources}

In addition to the iCOPE with COVID-19 intervention protocol, parents and caregivers were provided with two additional resources to support child mental well-being. First, an information sheet with basic information on promoting optimal sleep habits and behaviors was provided. Second, an information sheet with strategies for parents to support their children was also provided. The parenting sheet included information on using age-appropriate and copingfocused language, minimizing exposure to media and adult conversations, maintaining regular routines and expectations when possible, adjusting expectations during extraordinary times, and engaging in self-compassion and self-care.

\section{Measures}

\section{Treatment Feasibility and Acceptability}

In line with previous research (Rojas et al., 2021), feasibility was operationalized as successful referral and retention of participants to the treatment protocol. We also evaluated whether technical failures had an impact on the feasibility of implementing the intervention. Acceptability of the protocol was indexed by satisfaction questionnaires that were sent to the child participant (i.e., the child satisfaction questionnaire or the adolescent satisfaction questionnaire) and their parent/caregiver (i.e., the parent satisfaction questionnaire). The satisfaction questionnaires consisted of 10 multiple choice questions, assessing therapeutic rapport, use of the manual, learning of new skills, usefulness of online sessions and overall impressions of support 
received through the protocol. The child questionnaire asked children to rate the extent to which they agreed with each statement on a three-point scale (i.e., very true, kind of true, and not true). A five-point scale was used for adolescents and parents (i.e., agree, slightly agree, not agree, slightly disagree and disagree). Qualitative feedback was also solicited regarding the positive and negative aspects about the program and suggestions for improvement of the treatment protocol. Information was collected through the online survey software Qualtrics (https://www.qualtrics.com). Responses to the questionnaires were anonymized to encourage participant engagement.

\section{Anxiety Symptoms}

To measure the effectiveness of the protocol, children's symptoms of anxiety were assessed prior to commencing the iCOPE with COVID-19 treatment and after its completion. The Screen for Child Anxiety and Related Disorders (SCARED) was used to assess symptoms of anxiety (Birmaher et al., 1999). The SCARED was administered to both the primary parent/caregiver and the child. A total score was obtained to indicate children's overall level of anxiety. Scores on individual subscales (i.e., social anxiety, panic disorder/significant somatic symptoms, generalized anxiety disorder, separation disorder, and significant school avoidance) were also calculated.

\section{Statistical Analyses}

To measure effectiveness of the iCOPE with COVID-19 treatment, scores from the SCARED were examined. Data from participants that completed the SCARED at both data points (i.e., the pre- and post-intervention) were included in the corresponding analyses. This data was then compared using paired t-tests, to determine whether pre- and post-test scores significantly differed from one another.

\section{Results}




\section{Sample Characteristics}

At total of 27 children received the iCOPE with COVID-19 intervention. On average 67\% were female with a mean age of 9.56 years (range: 6- to 12-years-old). A total of 10 children completed in the outcome questionnaires and total of 8 parents completed the parent outcome questionnaires. Characteristics of the entire sample and the child and parent subsamples are reported in Table 1. A comparison on demographic characteristics indicated that there was no significant difference in age $(t=.60, p=.180)$ or sex (Chi-square $=.21, p=.648)$ for those who did and did not complete outcome measures.

(Insert table 1 about here)

\section{Treatment Feasibility and Acceptability}

There was high demand for the intervention as 44 families self-referred their children to participate. Of these, 27 youth received the iCOPE with COVID-19 protocol. Two children received the iCOPE with COVID-19 protocol but were continued to be seen for months afterwards due to severity of symptoms. For participants that received long term treatment, only data collected from the post-intervention measures following the three sessions of iCOPE with COVID-19 was included in this analysis. Only one child (3.7\% of total participants) did not complete all three sessions of the protocol and four children (14.8\% of total participants) received the intervention across 4 sessions, as opposed to three. With regards to treatment feasibility, all referring families had access to the technological infrastructure (i.e., electronic device and access to videoconferencing software) to participate in the intervention. There were no technological malfunctions and no sessions were cancelled or missed due to technological difficulties. All families were able to receive and access the electronic intervention manual. 
TELEMENTAL HEALTH FOR CHILDREN AND ADOLESCENTS DURING COVID-19 13

Regarding treatment acceptability, 10 children and 9 parents completed the client satisfaction questionnaires. See Supplementary Table 1 for detailed information regarding client responses to the questionnaires. Across all responses for youth and parents related to questions about protocol satisfaction and acceptability, 80-100\% of respondents selected "very true/agree", indicating an overall positive response to the protocol.

\section{Anxiety Symptoms}

To measure the effectiveness of the iCOPE with COVID-10 treatment, paired t-tests were conducted examining the pre- and post-intervention SCARED scores. In total, 10 children and 8 parents completed both the pre- and post-intervention questionnaires. The child questionnaires were completed on average 17.7 days (range: 0-40 days) after completing the protocol. Results from the child questionnaires demonstrated a significant decrease in the social anxiety subscale from pre- to post-intervention $(t(9)=3.37, p=.008, d=.1 .07)$. Trends towards a decrease in symptoms were also observed for the total anxiety score $(t(9)=2.30, p=.073, d=.64)$ and separation anxiety $(t(9)=1.87, p=.095, d=.59)$. Parent questionnaires were completed on average 17.1 days after their child's completion of the intervention (range: 1-40 days). No significant results were observed from the parent questionnaires. See Table 2 for detailed results on a pre- and post-intervention evaluation.

(Insert Table 2 about here)

\section{Discussion}

The COVID-19 pandemic and the associated public health restrictions have contributed to a significant rise in mental health concerns for children and adolescents around the world (Lee, 2020; Racine et al. 2020). As a result of school closures, uncertainty, and risk of illness, many children and adolescents have experienced increases in mental distress, including anxiety 
symptoms, that would benefit from an evidence-based intervention. At the outset of the pandemic, there were limited evidence-based interventions that could be used via telemental health with children and youth to address pandemic-related mental distress. Thus, the goal of the current investigation was to develop a brief, cognitive-behavioral, telemental health intervention (iCOPE with COVID-19) to address mental distress in children and adolescents during the COVID-19 pandemic. In addition to presenting the content and development of the intervention, we also provided preliminary evidence for the feasibility acceptability of the intervention developed as well as pilot evaluations of the effectiveness of the intervention. Two important findings emerged from the current study. First, based on preliminary evaluation data, the iCOPE with COVID-19 intervention was found to be feasible and acceptable to children and families who received the intervention. Second, we documented statistically significant decreases in self-reported social anxiety and marginally significant decreases in self-reported total anxiety, but not parent-reported anxiety, from pre to post-intervention. Taken together, the current evaluation is a first step in demonstrating the utility of the iCOPE with COVID-19 intervention for addressing child and adolescent mental distress related to the COVID-19 pandemic. A discussion of the current findings is expanded upon below.

An important outcome of the current study was that children/adolescents and their parents found the iCOPE with COVID-19 intervention to be feasible and acceptable. With regards to feasibility, consistent with previous telemental health investigations with children (Myers et al., 2013), participants successfully completed the three-session therapy course, and all participants had the technological infrastructure to participate in the intervention via online teleconferencing. Although access to technology and a reliable internet connection has been identified as a barrier in other investigations, the fact that the current study was conducted in a large urban centre in a 
country with generally high levels of access to a reliable internet connection likely contributed to the high feasibility in the current study. With regards to acceptability, children/adolescents and their parents generally identified that they found the intervention helpful, that they liked to content of the intervention, that they learned new tools, and that delivery online worked well. Importantly, children and parents also favourably endorsed items pertaining to the therapists' characteristics such as "being easy to talk to", "listened to the client", and "client's thoughts and worries were taken seriously". Prior work using telemental health interventions has highlighted the critical importance of being able to establish a positive therapeutic relationship with a client (Cook \& Doyle, 2004; Glueck, 2013; Goldstein \& Glueck, 2016;). Thus, the high levels of satisfaction with regards to the client and parent's relationship with the therapist in the current study may have played a role in some of the symptom improvements that were observed.

A promising finding from this small pilot study were observed mean decreases in symptoms of social anxiety and total anxiety as self-reported by children and adolescents after only three sessions. Given that iCOPE with COVID-19 was designed based on evidence-informed principles of cognitive-behavior therapy, which has a strong body of evidence to support its effectiveness, these findings are not entirely surprising, yet encouraging given the brief (three session) format of the intervention. Interestingly, these findings were only observed for the child/adolescent reports, not based on parent-reports. Previous research has demonstrated that inconsistencies among parent and child reports of mental health symptoms are common, especially for internalizing difficulties, such as anxiety (Hughes \& Gullone, 2020; Cole et al., 2000). As demonstrated in the current study, youth generally report higher levels of internalizing difficulties than their parents do (Cole et al., 2000), which may have made it easier to document a change or reduction in symptoms. It may be that parents had not yet observed over changes in anxious 
behaviors in their child or adolescent, while the child or adolescent themselves did experience a change in internal experiences of anxiety.

Though we found a statistically significant improvement in self-reported social anxiety and a trend towards a mean decrease in the total anxiety score, we did not find a significant change in other areas of anxiety that were assessed including separation anxiety, generalized anxiety, panic disorder, and school avoidance. This trend towards a mean decrease in total anxiety is in line with the goals of the intervention which were to provide children with psychoeducation and strategies for managing anxiety related to COVID-19, rather than to address specific anxiety disorders. Additionally, it is possible that pandemic related changes may have impacted areas of concern regarding anxiety for children and adolescents. For example, due to the pandemic, schools were closed and school avoidance related anxiety may not have been relevant while participating in this intervention.

Though this intervention was not designed to specifically target social anxiety, it was informed by cognitive-behavioural therapy, which is an effective evidence-based treatment for social anxiety (Scaini et al., 2016) and this intervention incorporated general skills that may be included in social anxiety treatment. Additionally, the onset of the COVID-19 pandemic resulted in significant disruptions of children's social lives. Indeed, emerging research has found that children reported fewer social contacts and a significant impairment in their relationships with friends during the pandemic (Ravens-Sieberer et al., 2021). Thus, it is possible that worries about social aspects of life may have been particularly relevant to children and adolescents during the intervention.

\section{Limitations and Future Directions}


Several limitations should be considered when interpreting the results. Due to its small sample size, the study was likely underpowered to detect statistically significant changes in other subscales of the SCARED measure. Therefore, replication of this pilot with a larger sample size is an important future research avenue. The nature of the study (i.e., a brief intervention with no follow-up outcome assessment), also did not allow us to investigate whether there were any longterm effects of the intervention or to evaluate whether the achieved treatment outcomes were maintained over time. Thus, determining the long-term effects of this intervention is an important area for future research. It may also be beneficial to include measures of other areas of psychosocial wellbeing, in addition to anxiety, such as measures of mood, quality of life, and social functioning, in future evaluations of this intervention. While the 3 -session format of the protocol allowed for greater accessibility to the community, it is currently unknown whether providing this intervention over a longer period of time (e.g., 6 sessions vs. 3) would increase effectiveness. Furthermore, the current study did not recruit a control group and thus we cannot assume that improvements in child anxiety symptoms were directly associated with the implementation of the intervention. Future work would benefit from including a comparison or wait-list control group. Lastly, this intervention was administered exclusively by doctoral level trainees, and therefore its effectiveness when delivered by other mental health professionals is currently unknown. Future research is warranted to determine whether iCOPE with COVID-19 could be delivered by diverse health care professionals or whether the intervention protocol could be adapted to be selfadministered by children and adolescents, and their parents. This is an important direction for further research, as this would greatly increase both the availability and accessibility of this intervention for children and their families.

\section{Conclusions}


The current study suggests that a short, 3-session, cognitive-behavioral intervention can be successfully delivered to children, and adolescents to address anxiety associated with the COVID19 pandemic. Additionally, there is some early evidence that a brief intervention that targets thinking, behavioral, and affective coping tools can help to reduce anxiety symptoms in children and adolescents, even after a brief intervention. Future work would benefit from a larger study sample to increase power to detect differences from pre to post-treatment as well as a longer follow-up period to identify whether treatment effects were maintained over time. 


\section{References}

Albano, A. M., \& Kendall, P. C. (2002). Cognitive behavioural therapy for children and adolescents with anxiety disorders: Clinical research advances. International Review of psychiatry, 14(2), 129-134.

Beck, A. T. (1979). Cognitive therapy and the emotional disorders. New American Library.

Birmaher, B., Brent, D. A., Chiappetta, L., Bridge, J., Monga, S., \& Baugher, M. (1999). Psychometric properties of the Screen for Child Anxiety Related Emotional Disorders (SCARED): A replication study. Journal of the American Academy of Child \& Adolescent Psychiatry, 38(10), 1230-1236.

Boydell, K. M., Hodgins, M., Pignatiello, A., Teshima, J., Edwards, H., \& Willis, D. (2014). Using technology to deliver mental health services to children and youth: a scoping review. Journal of the Canadian Academy of Child and Adolescent Psychiatry, 23(2), 87.

Calear, A. L., \& Christensen, H. (2010). Review of internet-based prevention and treatment programs for anxiety and depression in children and adolescents. Medical Journal of Australia, 192, S12-S14.

Christensen, H., Griffiths, K., Groves, C., \& Korten, A. (2006). Free range users and one hit wonders: community users of an Internet-based cognitive behaviour therapy program. Australian \& New Zealand Journal of Psychiatry, 40(1), 59-62.

Cole, D. A., Hoffman, K., Tram, J. M., \& Maxwell, S. E. (2000). Structural differences in parent and child reports of children's symptoms of depression and anxiety. Psychological assessment, 12(2), 174.

Cook, J. E., \& Doyle, C. (2002). Working alliance in online therapy as compared to face-to-face therapy: Preliminary results. CyberPsychology \& Behavior, 5(2), 95-105. 
TELEMENTAL HEALTH FOR CHILDREN AND ADOLESCENTS DURING COVID-19 20

Cost, K. T., Crosbie, J., Anagnostou, E., Birken, C. S., Charach, A., Monga, S., ... \& Korczak, D. J. (2021). Mostly worse, occasionally better: impact of COVID-19 pandemic on the mental health of Canadian children and adolescents. European Child \& Adolescent Psychiatry, 1-14.

Goldstein, F., \& Glueck, D. (2016). Developing rapport and therapeutic alliance during telemental health sessions with children and adolescents. Journal of Child and Adolescent Psychopharmacology, 26(3), 204-211.

Glueck, D., Myers, K., \& Turvey, C. L. (2013). Establishing therapeutic rapport in telemental health. Telemental health: Clinical, technical and administrative foundations for evidence-based practice, 29-46.

Hollis, C., Falconer, C. J., Martin, J. L., Whittington, C., Stockton, S., Glazebrook, C., \& Davies, E. B. (2017). Annual Research Review: Digital health interventions for children and young people with mental health problems-a systematic and meta-review. Journal of Child Psychology and Psychiatry, 58(4), 474-503.

Jiao, W. Y., Wang, L. N., Liu, J., Fang, S. F., Jiao, F. Y., Pettoello-Mantovani, M., \& Somekh, E. (2020). Behavioral and emotional disorders in children during the COVID-19 epidemic. The Journal of Pediatrics, 221, 264.

Law, E. F., Murphy, L. K., \& Palermo, T. M. (2012). Evaluating treatment participation in an internet-based behavioral intervention for pediatric chronic pain. Journal of pediatric psychology, 37(8), 893-903.

Lee, J. (2020). Mental health effects of school closures during COVID-19. The Lancet Child \& Adolescent Health, 4(6), 421. 
Liu, W., Zhang, Q. I., Chen, J., Xiang, R., Song, H., Shu, S., ... \& Liu, Y. (2020). Detection of Covid-19 in children in early January 2020 in Wuhan, China. New England Journal of Medicine, 382(14), 1370-1371.

Ma, X., Yue, Z. Q., Gong, Z. Q., Zhang, H., Duan, N. Y., Shi, Y. T., ... \& Li, Y. F. (2017). The effect of diaphragmatic breathing on attention, negative affect and stress in healthy adults. Frontiers in Psychology, 8, 874.

Madigan, S., Racine, N., Cooke, J. E., \& Korczak, D. J. (2020). COVID-19 and telemental health: Benefits, challenges, and future directions. Canadian Psychology/Psychologie canadienne.

March, S., Spence, S. H., \& Donovan, C. L. (2008). The efficacy of an internet-based cognitivebehavioral therapy intervention for child anxiety disorders. Journal of Pediatric Psychology, 34(5), 474-487.

McLoone, J., Hudson, J. L., \& Rapee, R. M. (2006). Treating anxiety disorders in a school setting. Education and Treatment of Children, 219-242.

Merry, S. N., Stasiak, K., Shepherd, M., Frampton, C., Fleming, T., \& Lucassen, M. F. (2012). The effectiveness of SPARX, a computerised self help intervention for adolescents seeking help for depression: randomised controlled non-inferiority trial. $B M J, 344$.

Myers, K., Stoep, A. V., \& Lobdell, C. (2013). Feasibility of conducting a randomized controlled trial of telemental health with children diagnosed with attention-deficit/hyperactivity disorder in underserved communities. Journal of Child and Adolescent Psychopharmacology, 23(6), 372-378.

Perepletchikova, F., Axelrod, S. R., Kaufman, J., Rounsaville, B. J., Douglas-Palumberi, H., \& Miller, A. L. (2011). Adapting dialectical behaviour therapy for children: Towards a new 
research agenda for paediatric suicidal and non-suicidal self-injurious behaviours. Child and Adolescent Mental Health, 16(2), 116-121.

Podell, J. L., Mychailyszyn, M., Edmunds, J., Puleo, C. M., \& Kendall, P. C. (2010). The Coping Cat Program for anxious youth: The FEAR plan comes to life. Cognitive and Behavioral Practice, 17(2), 132-141.

Racine, N., Cooke, J. L., Eirich, R., Korczak, D. J., McArthur, B., \& Madigan, S. (2020). Child and adolescent mental illness during COVID-19: A rapid review. Psychiatry Research.

Rapee, R. M., Wignall, A., Hudson, J. L., \& Schniering, C. A. (2000). Evidence-based treatment of child and adolescent anxiety disorders. New Harbinger.

Ravens-Sieberer, U., Kaman, A., Erhart, M., Devine, J., Schlack, R., \& Otto, C. (2021). Impact of the COVID-19 pandemic on quality of life and mental health in children and adolescents in Germany. European Child \& Adolescent Psychiatry, 1-11.

Rojas, S. M., Gold, S. D., Bryan, C. J., Pruitt, L. D., Felker, B. L., \& Reger, M. A. (2021). Brief Cognitive-Behavioral Therapy for Suicide Prevention (BCBT-SP) via Video Telehealth: A Case Example During the COVID-19 Outbreak. Cognitive and Behavioral Practice

Rosen, Z., Weinberger-Litman, S. L., Rosenzweig, C., Rosmarin, D. H., Muennig, P., Carmody, E. R., ... \& Litman, L. (2020). Anxiety and distress among the first community quarantined in the US due to COVID-19: Psychological implications for the unfolding crisis. PsyArXiv. doi: 10.31234/osf.io/7eq8c

Scaini, S., Belotti, R., Ogliari, A., \& Battaglia, M. (2016). A comprehensive meta-analysis of cognitive-behavioral interventions for social anxiety disorder in children and adolescents. Journal of Anxiety Disorders, 42, 105-112. 
Smirni, P., Lavanco, G., \& Smirni, D. (2020). Anxiety in Older Adolescents at the Time of COVID-19. Journal of Clinical Medicine, 9(10), 3064.

Stinson, J. N., Lalloo, C., Harris, L., Isaac, L., Campbell, F., Brown, S., ... \& Karim, A. (2014). iCanCope with Pain ${ }^{\mathrm{TM}}$ : user-centred design of a web-and mobile-based self-management program for youth with chronic pain based on identified health care needs. Pain Research and Management, 19(5), 257-265.

Ye, X., Bapuji, S. B., Winters, S. E., Struthers, A., Raynard, M., Metge, C., ... \& Sutherland, K. (2014). Effectiveness of internet-based interventions for children, youth, and young adults with anxiety and/or depression: A systematic review and meta-analysis. $B M C$ Health Services Research, 14(1), 1-9.

Xie, X., Xue, Q., Zhou, Y., Zhu, K., Liu, Q., Zhang, J., \& Song, R. (2020). Mental health status among children in home confinement during the coronavirus disease 2019 outbreak in Hubei Province, China. JAMA Pediatrics, 174(9), 898-900.

Zhou, S. J., Zhang, L. G., Wang, L. L., Guo, Z. C., Wang, J. Q., Chen, J. C., ... \& Chen, J. X. (2020). Prevalence and socio-demographic correlates of psychological health problems in Chinese adolescents during the outbreak of COVID-19. European Child and Adolescent Psychiatry, 29(6), 749-758. 


\section{Table 1}

Sample Characteristics

\begin{tabular}{|l|c|c|c|c|}
\hline & Overall Sample & Sample Included in & Sample Included in & Sample Who Did \\
& & Child Outcome & Parent Outcome & Not Complete \\
& & Analysis & Analysis & Child Outcome \\
& $(N=27)$ & $(n=10)$ & $(n=8)$ & Measures \\
\cline { 2 - 5 } & $67 \%$ & $60 \%$ & $63 \%$ & $65 \%$ \\
\hline Female & $9.56(1.50)$ & $10.10(1.37)$ & $9.63(1.58)$ & $9.25(1.52)$ \\
\hline Age & & & & \\
\hline
\end{tabular}


Table 2

Pre-and Post-intervention Anxiety Measurement

\begin{tabular}{|l|l|l|l|l|l|}
\hline & $\begin{array}{l}\text { Pre-Intervention } \\
\text { M (SD) }\end{array}$ & $\begin{array}{l}\text { Post-Intervention } \\
\text { M (SD) }\end{array}$ & $t$ & $p$ & $\mathrm{~d}$ \\
\hline Child/Youth & & & & \\
\hline Total anxiety & $24.75(12.38)$ & $19.30(13.06)$ & 2.03 & .073 & .64 \\
\hline Social anxiety & $6.20(2.53)$ & $4.10(2.08)$ & 3.37 & .008 & 1.07 \\
\hline Panic disorder & $5.10(4.48)$ & $4.50(5.70)$ & .50 & .630 & .16 \\
\hline Generalized anxiety & $5.30(4.45)$ & $4.20(3.46)$ & .99 & .348 & .31 \\
\hline Separation anxiety & $6.10(2.77)$ & $4.30(3.68)$ & 1.87 & .095 & .59 \\
\hline School avoidance & $2.10(2.42)$ & $1.90(2.33)$ & .514 & .619 & .16 \\
\hline Parent & & & & & \\
\hline Total anxiety & $18.12(10.37)$ & $18.00(10.89)$ & .04 & .967 & .02 \\
\hline Social anxiety & $4.50(4.14)$ & $4.63(3.42)$ & -.36 & .732 & .13 \\
\hline Panic disorder & $1.88(1.46)$ & $1.75(1.91)$ & .15 & .882 & .05 \\
\hline Generalized anxiety & $6.00(4.41)$ & $5.00(4.28)$ & .83 & .436 & .29 \\
\hline Separation anxiety & $4.25(3.28)$ & $4.50(3.30)$ & -.31 & .769 & .11 \\
\hline School avoidance & $1.50(1.20)$ & $1.75(2.19)$ & -.40 & .699 & .14 \\
\hline & & & & & \\
\hline
\end{tabular}




\section{Supplemental Materials}

\section{Supplementary Table 1}

Treatment Acceptability and Feasibility Questionnaire Responses

\begin{tabular}{|c|c|c|c|c|c|c|}
\hline \multirow[t]{2}{*}{ Item } & \multicolumn{2}{|c|}{$\begin{array}{l}\text { Very True* } \\
\text { Agree** }\end{array}$} & \multicolumn{2}{|c|}{$\begin{array}{l}\text { Kind of True* } \\
\text { Slightly } \\
\text { Agree** }\end{array}$} & \multicolumn{2}{|c|}{$\begin{array}{l}\text { Not True* } \\
\text { Not agree, slightly } \\
\text { disagree or disagree** }\end{array}$} \\
\hline & Youth & Parent & Youth & Parent & Youth & Parent \\
\hline Therapist was easy to talk to & $80 \%$ & $100 \%$ & $20 \%$ & $0 \%$ & $0 \%$ & $0 \%$ \\
\hline Therapist listened to client & $100 \%$ & $100 \%$ & $0 \%$ & $0 \%$ & $0 \%$ & $0 \%$ \\
\hline $\begin{array}{l}\text { Client's thoughts and worries } \\
\text { were taken seriously }\end{array}$ & $80 \%$ & $100 \%$ & $20 \%$ & $0 \%$ & $0 \%$ & $0 \%$ \\
\hline $\begin{array}{l}\text { Meetings with therapist were } \\
\text { helpful }\end{array}$ & $100 \%$ & $92 \%$ & $0 \%$ & $8 \%$ & $0 \%$ & $0 \%$ \\
\hline $\begin{array}{l}\text { Client felt comfortable sharing } \\
\text { thoughts and feelings with } \\
\text { therapist }\end{array}$ & $80 \%$ & $92 \%$ & $20 \%$ & $8 \%$ & $0 \%$ & $0 \%$ \\
\hline $\begin{array}{l}\text { Liked the iCOPE manual or other } \\
\text { worksheets }\end{array}$ & $80 \%$ & $100 \%$ & $20 \%$ & $0 \%$ & $0 \%$ & $0 \%$ \\
\hline Client learned new things/skills & $100 \%$ & $92 \%$ & $0 \%$ & $8 \%$ & $0 \%$ & $0 \%$ \\
\hline $\begin{array}{l}\text { The online meetings worked fine } \\
\text { for the client }\end{array}$ & $90 \%$ & $83 \%$ & $10 \%$ & $8 \%$ & $0 \%$ & $8 \%$ \\
\hline
\end{tabular}




\begin{tabular}{|c|c|c|c|}
\hline Item & $\begin{array}{l}\text { Very True* } \\
\text { Agree** }\end{array}$ & $\begin{array}{l}\text { Kind of True* } \\
\text { Slightly } \\
\text { Agree** }\end{array}$ & $\begin{array}{l}\text { Not True* } \\
\text { Not agree, slightly } \\
\text { disagree or disagree** }\end{array}$ \\
\hline $\begin{array}{l}\text { If a friend needed similar help, } \\
\text { they would tell them about the } \\
\text { program }\end{array}$ & $100 \%$ & $20 \%$ & $0 \%$ \\
\hline $\begin{array}{l}\text { Overall, the help the client } \\
\text { received was good }\end{array}$ & $100 \%$ & $0 \% \quad 0 \%$ & $0 \%$ \\
\hline
\end{tabular}

Note. Percentage of responses indicated on client satisfaction questionnaires. Youth responses represent responses on both the child and adolescent satisfaction questionnaires. Responses that were marked as not agree, slightly disagree or disagree on the adolescent or parents form were combined together for reporting purposes.

* As presented on the Child Satisfaction Questionnaire

** As presented on the Adolescent and Parent Satisfaction Questionnaires 
Figure 1

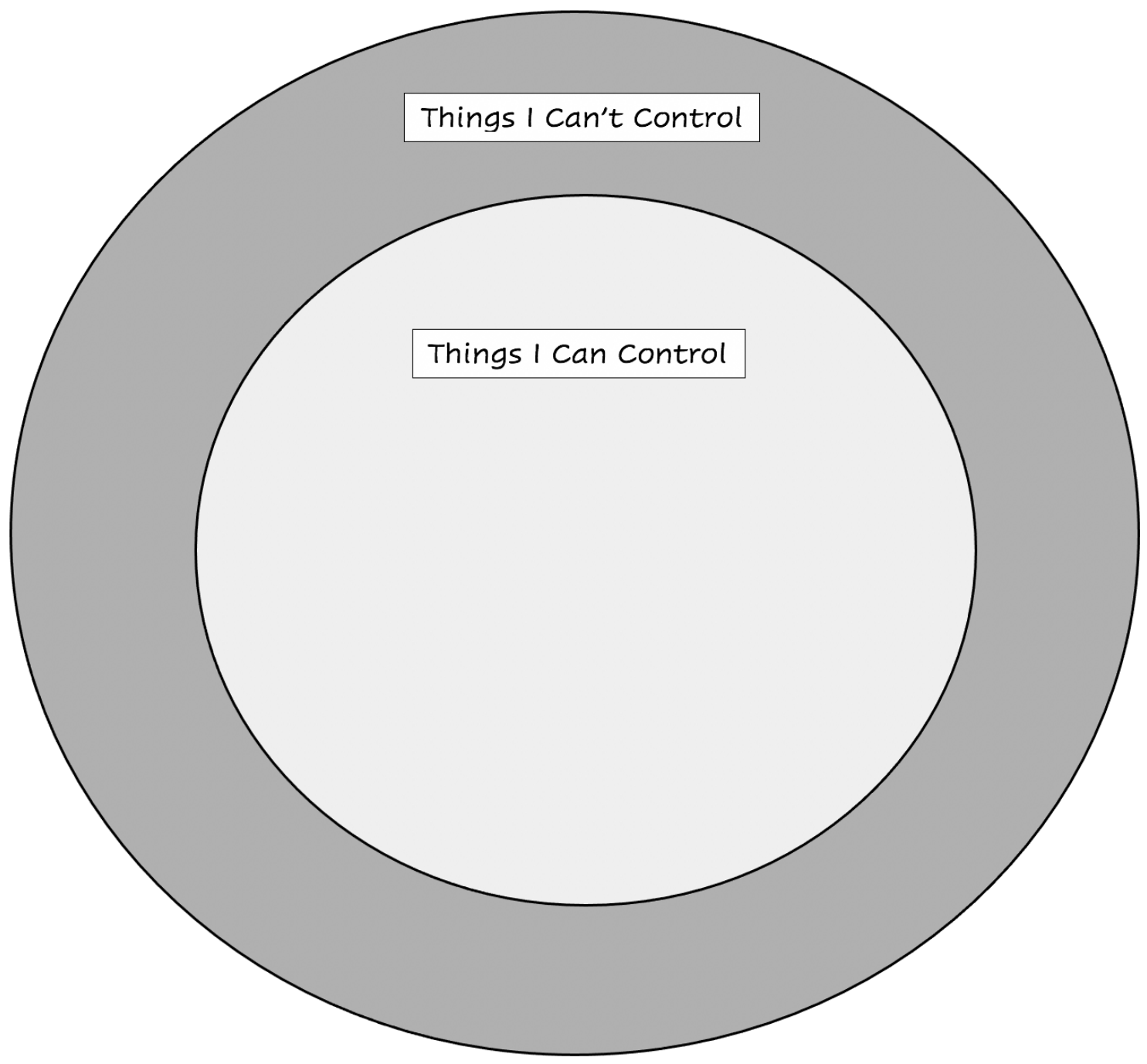

Figure 1. Circle of control activity. 